Kerstin Lebahn*, Kerstin Stöffler, Stefan Oschatz, Daniela Arbeiter, Thomas Reske, KlausPeter Schmitz, Gerrit Paasche, Thomas Lenarz and Niels Grabow

\title{
Polymer selection for Eustachian tube stent application based on mechanical, thermal and degradation behavior
}

\begin{abstract}
The novel concept of stenting the Eustachian tube was established to provide an effective and safe therapy of Eustachian tube dysfunction. Biodegradable polymer stents are being developed to restore impaired tube function. As the supporting effect may be required for different time periods, PLA-co-PEG copolymers, PLLGA, PDLLA and PDS, having shorter degradation times compared to PLLA, were evaluated as potential stent materials. Since tensile tests and thermal analyses of solvent cast films from PLA-co-PEG copolymers showed comparable properties to PLLA, stent samples were manufactured from these materials. Mechanical stent testing revealed an increase of elastic recoil and slight decrease of collapse pressure compared to PLLA. In a short term accelerated degradation study a considerable percentage molar mass reduction and an increasing degree of crystallinity depending on PEG content was found. Based on the results obtained, the tested polymers offer a promising, faster degradable alternative to the established stent material PLLA.
\end{abstract}

Keywords: Eustachian tube dysfunction, polymeric stent, degradation time, PLA-co-PEG copolymers.

https://doi.org/10.1515/cdbme-2021-2167

\section{Introduction}

Blockage of the Eustachian tube isolates the middle ear from the outside environment leading to acute otitis media, otitis media with effusion or chronic otitis media. Eustachian tube dysfunction (ETD) with insufficient ventilation of the middle ear can induce reversible functional problems but is also main cause of permanent conductive hearing loss [1]. Prevalence of chronic ETD is about $1 \%$ of adult population [2]. Children up to the age of 10 years even develop at least a passive disorder of Eustachian tube function in about $40 \%$ [3]. The innovative concept of stenting the Eustachian tube offers the potential to become an effective minimal-invasive therapeutic option for the treatment of ETD.

As described in [4], biodegradable polymeric stents are being developed to restore impaired tube function and dissolve after fulfilling their supportive purpose. Stents made of poly(L-lactide) (PLLA) were fabricated and tested in human cadaver studies [5] and are currently being investigated in in vivo animal studies. However, depending on particular pathophysiology, supporting effect of stents may be required for different periods of time. Therefore, in the current study, alternative polymers with shorter degradation time were evaluated as potential stent materials in comparison to PLLA.

\section{Materials and methods}

\subsection{Manufacturing of test samples}

To make a preselection for stent fabrication, solvent cast films of the polymers listed in Table 1 (all polymers purchased from Evonik Industries AG, Essen, Germany) were manufactured from chloroform solutions: $1 \mathrm{~g}$ polymer in $24 \mathrm{ml}$ chloroform.
ImplantTechnology and Biomaterials e.V., Rostock, Germany

Gerrit Paasche, Thomas Lenarz: Department of Otorhino-

laryngology, Hannover Medical School, Hannover, Germany 
Solution casted tubes for stent manufacturing (ID $=1.4 \mathrm{~mm}$, $\mathrm{WT}=150 \mu \mathrm{m})$ were fabricated in an automated dip-coating process with subsequent washing and drying for solvent removal, according to [6]. Selected polymers showing mechanical and thermal properties comparable to PLLA in conjunction with a good surface morphology in preliminary tests were used: LP t 16, LP t 46, LG 857 S, R 207 S as well as $\mathrm{L} 210 \mathrm{~S}$ as reference. Since satisfactory tube quality could not be achieved with LG 857 S and R 207 S, stent prototypes were fabricated from the poly(L-lactide)-co-poly(ethylene glycol) (PLA-co-PEG) copolymers LP t 16 and LP t 46 as well as PLLA L $210 \mathrm{~S}$ in a $\mathrm{CO}_{2}$ laser cutting process. Half of the stents were subjected to a thermo-mechanical treatment, according to [7], to enable smooth expandability.

Table 1: Composition and degradation timeframe of polymers examined [8]

\begin{tabular}{lll}
\hline Polymer name & Composition & $\begin{array}{l}\text { Degradation } \\
\text { timeframe }\end{array}$ \\
\hline RESOMER L 210 S & Poly(L-lactide) (PLLA) & $>3$ years \\
RESOMER LG 857 S & $\begin{array}{l}\text { Poly(L-lactide-co- } \\
\text { glycolide) 85:15 } \\
\text { (PLLGA) }\end{array}$ & $1-2$ years \\
RESOMER R 207 S & $\begin{array}{l}\text { Poly(D,L-lactide) } \\
\text { (PDLLA) }\end{array}$ & $1-2$ years \\
RESOMER LP t 16 & $\begin{array}{l}\text { Poly(-lactide-co-PEG) } \\
\text { triblock (PLA-co-PEG) }\end{array}$ & $<12$ months \\
RESOMER LP t 46 & $\begin{array}{l}\text { Poly(L-lactide-co-PEG) } \\
\text { triblock (PLA-co-PEG) }\end{array}$ & $<7$ months \\
RESOMER X 206 S & Polydioxanone (PDS) & $<6$ months \\
\hline
\end{tabular}

\subsection{Analysis of cast films and tubes}

\subsubsection{Uniaxial tensile testing}

Tensile test specimens with standard specimen geometry $1 \mathrm{BB}$ according to DIN EN ISO 527 were punched from the cast foils. Thickness of each test specimen was measured at 3 positions using Mitutoyo Absolute digital indicator (Mitutoyo Corp, Tokyo, Japan). Uniaxial tensile tests were performed with a universal testing machine zwickiLine Z2.5 (type BZ2.5/TN 1S, Zwick/Roell, Ulm, Germany) with a $50 \mathrm{~N}$ load cell. Tests were carried out at $37{ }^{\circ} \mathrm{C}$ with a crosshead speed of $25 \mathrm{~mm} / \mathrm{min}$. Ten specimens of each material were tested. Young's modulus was calculated in the linear-elastic strain region. Besides, tensile strength and elongation at break were compared for tested polymers.

\subsubsection{Thermal and morphological characterization}

Melting behavior of the solvent cast samples was measured using a DSC 1 system (Mettler Toledo, Switzerland) with a heating rate of $10 \mathrm{~K} / \mathrm{min}$. The data were analyzed in terms of glass transition $\left(\mathrm{T}_{\mathrm{G}}\right)$, melting temperature $\left(\mathrm{T}_{\mathrm{M}}\right)$ and enthalpy of transition $(\Delta \mathrm{H})$. Only one sample of each material was examined to obtain a general insight into thermal properties.

Scanning electron microscopy (SEM) was carried out using a Quattro S (Thermo Fisher Scientific, Waltham, USA) in low vacuum mode at $5 \mathrm{kV}$ to analyze the surface morphology of solvent cast films as well as solution casted tubes. Images were taken with a magnification of 500x.

To determine the wall thickness of polymer tubes after the washing and drying process cross-sectional images are generated with the micro CT scanner SkyScan 1172 (Bruker, Kontich, Belgium). All samples were scanned without filters with source voltage of $40 \mathrm{kV}$ and $100 \mu \mathrm{A}$ current and a resolution of $4.8 \mu \mathrm{m}$.

\subsection{Mechanical stent testing}

Testing of stent samples was performed according to DIN EN ISO 25539-2 using a dedicated test setup, according to [9]. In a temperature controlled water bath $\left(37^{\circ} \mathrm{C}\right)$, the balloon was inflated by a computer-controlled pump to nominal diameter of $3.0 \mathrm{~mm}$. Stent profile during expansion and stent recoil was measured optically with a two-axis laser scanner (ODAC 64 XY, Zumbach Electronic AG, Orpund, Switzerland).

For analysis of radial strength the stent samples were implanted in thin polyurethane tubes fixed into a sealed test chamber, according to [10], which was filled with temperature controlled water $\left(37^{\circ} \mathrm{C}\right)$ and connected to a computer driven pressure control unit. Hydraulic pressure in the test chamber was incrementally increased in steps of $2 \mathrm{kPa}$ to apply a radial force on the tube around the stent. The pressure at which the stent loses its support function was noted as collapse pressure. A total of 10 untreated and 10 thermomechanical treated stents of each polymer were studied.

\subsection{Accelerated degradation study}

\subsubsection{Degradation conditions}

In a first short term accelerated degradation study stent samples were degraded for 6 weeks in $2 \mathrm{ml}$ Sørensen buffer at $\mathrm{pH}=7.38$ (Morphisto, Frankfurt/Main, Germany) at $55^{\circ} \mathrm{C}$ and shaken with $100 \mathrm{rpm}$. Buffer solution was changed every 
week. Five samples of each polymer were investigated after 2, 4, and 6 weeks, respectively, after being washed 3 times with distilled water and dried in vacuo at $40{ }^{\circ} \mathrm{C}$ for 1 week.

\subsubsection{Characterization of degraded samples}

Sample masses were identified prior and after degradation using an electronic microbalance (XP6U, Mettler Toledo International, Inc., Zurich, Switzerland) to calculate percentage mass change.

Molecular weight was determined by GPC measurements using a PSS SECcurity SEC system (Polymer Standard Services GmbH, Mainz, Germany) with integrated RI detector combined with a viscosity detector (WEG Dr. Bures GmbH, Dallgow, Germany). Molecular weights were identified by the universal calibration procedure using twelve polystyrene calibration standards ranging from 376 to 2,570,000 g/mol.

Thermal (DSC) and surface morphological (SEM) analyses of the degraded stent samples were performed using the same methods as for cast films and tubes described above.

\section{Results}

\subsection{Mechanical and thermal properties of solvent cast films}

LP t 16 and LP t 46 show comparable mechanical properties to PLLA, although the elongation at break of LP t 46 is much higher and has a higher standard deviation (Figure 1). Young's modulus and tensile strength of LG 857 S and R 207 are substantially reduced.

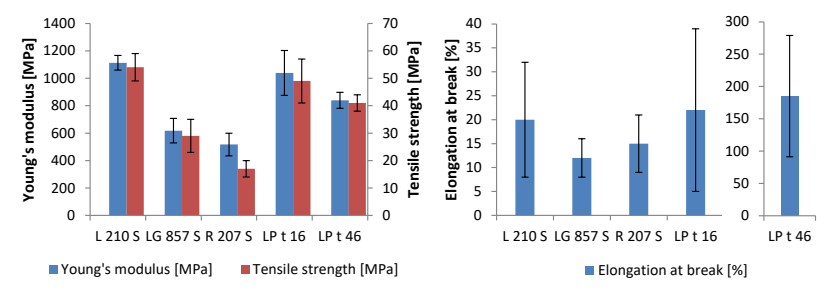

Figure 1: Young's modulus, tensile strength and elongation at break of solvent cast films determined with tensile tests

In DSC analyses LP t 16 and LP t 46 show similar values to PLLA, with the glass transition and melting temperature as well as transition enthalpy decreasing slightly with increasing PEG content (Table 2). Due to its completely amorphous structure, R 207 has no melting point.
Table 2: Glass transition $\left(T_{G}\right)$, melting temperature $\left(T_{M}\right)$ and transition enthalpy $(\Delta \mathrm{H})$ of solvent cast films measured with DSC

\begin{tabular}{lccl}
\hline Polymer & $\mathbf{T}_{\mathbf{G}}\left[{ }^{\circ} \mathbf{C}\right]$ & $\mathbf{T}_{\mathbf{M}}\left[{ }^{\circ} \mathbf{C}\right]$ & $\Delta \mathbf{H}[\mathbf{J} / \mathbf{g}]$ \\
\hline RESOMER L 210 S & 65.63 & 178.52 & 40.31 \\
RESOMER LG 857 S & 65.79 & 150.93 & -1.18 \\
RESOMER R 207 S & 62.07 & - & - \\
RESOMER LP t 16 & 63.42 & 176.86 & 35.32 \\
RESOMER LP t 46 & 61.67 & 175.31 & 35.95 \\
RESOMER X 206 S & -10.62 & 106.16 & 97.64 \\
\hline
\end{tabular}

\subsection{Morphological structure of solvent cast films and tubes}

Since X 206 S showed a porous structure of the cast films, it was not considered for further investigations. Due to the high shrinkage during the washing/drying process, tubes made of LG 857 showed very irregular wall thicknesses. The tubes made of R 207 did not dry completely owing to their amorphous structure and could thus not be removed well from the dipping mandrel. Cast films or dip-coated tubes made of L 210 S, LP t 16 and LP t 46 showed homogeneous surface structures in SEM and uniform wall thicknesses in micro-CT cross-sectional studies, so stents were subsequently fabricated from these polymers.

\subsection{Mechanical properties of stent samples}

As all untreated stents showed strut ruptures or at least incipient cracks, recoil and collapse measurements could only be evaluated for 4, 1 and 4 stents with cracks of L 210 $\mathrm{S}$, LP t 16 and LP t 46, respectively, which results in unreliable measuring values and high standard deviations (Figure 2). Compared to PLLA, treated samples of LP t 16 and LP t 46 show an increase of elastic recoil from $2.0 \%$ to $3.4 \%$ and $3.2 \%$ and decrease of collapse pressure from 1.0 bar to 0.7 bar and 0.8 bar, respectively.

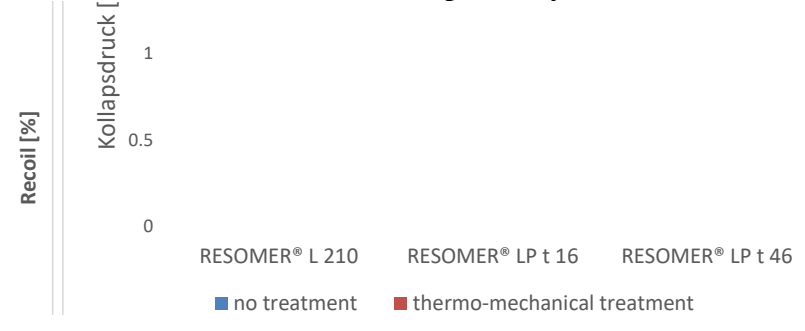

Figure 2: Elastic recoil and collapse pressure of stent samples without and with thermo-mechanical treatment 


\subsection{Influence of degradation on material properties}

Mass changes linearly for all three materials tested to approx. $96 \%$ after 6 weeks of accelerated degradation (Figure 3). Molecular weight is reduced to about $60 \%, 40 \%$ and $25 \%$ from the initial value for L $210 \mathrm{~S}$ (initially approx. $290 \mathrm{kDa}$ ), LP t $16(590 \mathrm{kDa})$ and LP t $46(210 \mathrm{kDa})$, respectively.

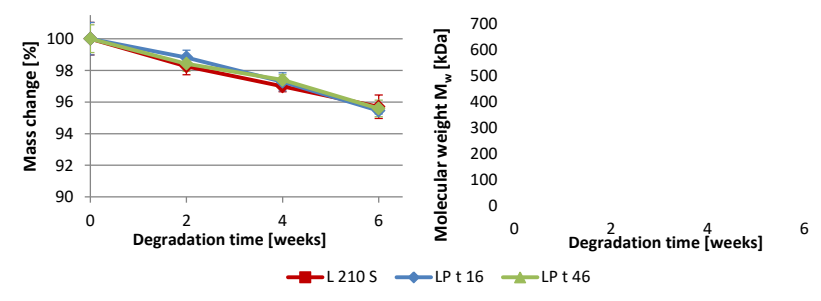

Figure 3: Percentage change in mass and molecular weight due to degradation

As degradation progressed, all three polymers investigated showed an increase in crystallinity with increasing PEG content in DSC analyses. Surface morphology investigated with SEM offers no optically detectable polymer degradation as the main mechanism is bulk degradation.

\section{Discussion}

Some polymers were excluded due to their difficult processability or insufficient surface quality. Adjustment of manufacturing processes could help to obtain suitable results. Although stent samples of LP t 16 and LP t 46 show a higher elastic recoil and decreasing collapse pressure compared to PLLA, values are still within an acceptable range for Eustachian tube stent application. The results of accelerated degradation give a first impression on the change of material properties in the initial phase but have to be further investigated to obtain information about complete decomposition or for example the resulting radial force of stents in the course of degradation.

\section{Conclusion}

Due to the obtained results, tested PLA-co-PEG copolymers offer a promising faster degrading alternative to the wellestablished stent material PLLA. Additional studies, for example on biocompatibility of stent materials and degradation products, as well as implantation tests need to be carried out to further confirm suitability for Eustachian tube stent application.

\section{Author Statement}

Research funding: This work was partially funded by the Federal Ministry of Education and Research (BMBF) within the project RESPONSE "Partnership for Innovation in Implant Technology". Conflict of interest: Authors state no conflict of interest. Informed consent: Informed consent is not applicable. Ethical approval: The conducted research is not related to either human or animals use.

\section{References}

[1] Lenarz T, Wilfling T, Paasche G, Schümann K, Grabow N, Schmidt W, Schmitz K-P, Müller H. Stenting the Eustachian Tube for Treatment of Chronic Otitis Media. Abstract, BMT 2018 - 52nd Annual Conference of the German Society for Biomedical Engineering, Aachen. Biomedical Engineering/ Biomedizinische Technik. 2018;63(s1)75-83.

[2] Schröder S, Reineke U, Lehmann M, Ebmeyer J, Sudhoff H. Chronic obstructive eustachian tube dysfunction in adults: Long-term results of balloon dilatation eustachian tuboplasty. HNO. 2013;61(2), 142-151.

[3] Di Martino E, Thaden R, Krombach GA, Westhofen M. Function tests for the Eustachian tube. Current knowledge. HNO. 2004;52(11),1029-1040.

[4] Schümann K, Wilfling T, Paasche G, Schuon R, Schmidt W, Müller H, Momma C, Schmitz K-P, Lenarz T, Grabow N. Development of biodegradable stents for the treatment of Eustachian tube dysfunction. Current Directions in Biomedical Engineering. 2018;4(1), 505-508.

[5] Schümann K, Wilfling T, Paasche G, Schuon R, Tautorat C, Schmitz K-P, Lenarz T, Grabow N. Polymeric stents for the Eustachian tube: development and human cadaver study. Current Directions in Biomedical Engineering. 6(3), 213-216.

[6] Grabow N, Bünger CM, Schultze C, Schmohl K, Martin DP, Williams SF, Sternberg K, Schmitz K-P. A biodegradable slotted tube stent based on poly(L-lactide) and poly(4hydroxybutyrate) for rapid balloon-expansion. Annals of biomedical engineering. 2007;35(12)2031-2038.

[7] Schümann K, Arbeiter D, Grabow N, Schmitz K-P. Method for the improvement of mechanical properties of biodegradable polymeric scaffolds. Current Directions in Biomedical Engineering. 2019;5(1)485-487.

[8] Evonik Industries AG. RESOMER® bioresorbable polymers for medical devices. healthcare.evonik.com/en/medicaldevices/biodegradable-materials/resomer-portfolio/standardpolymers (accessed: 21 june 2021)

[9] Schmidt W, Behrens P, Behrend D, Schmitz K. Measurement of Mechanical Properties of Coronary Stents according to the European Standard prEN 12006-3. Progress in Biomedical Research. 1999;1(4)45-51.

[10] Schmidt W, Behrens P, Brandt-Wunderlich C, Siewert S, Grabow N, Schmitz K-P: In vitro performance investigation of bioresorbable scaffolds - Standard tests for vascular stents and beyond, Cardiovasc. Revasc. Med. 17(2016) 375-383. 